\title{
Cognitive Function of Children and Adolescents with Attention- Deficit/Hyperactivity Disorder in a 2-Year Open-Label Study of Lisdexamfetamine Dimesylate
}

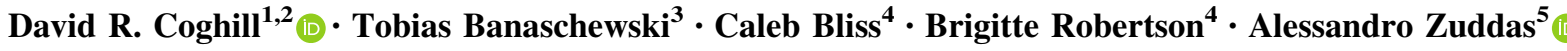

Published online: 30 January 2018

(C) Shire Development LLC 2018. This article is an open access publication

\begin{abstract}
Background SPD489-404 was the first 2-year safety study of lisdexamfetamine dimesylate in the treatment of attention-deficit/hyperactivity disorder in children and adolescents. In accordance with advice from the European Medicines Agency, assessment of cognitive function was a predefined safety outcome in SPD489-404.

Objective The objective of this study was to assess cognitive function over 2 years in study SPD489-404, using the Cambridge Neuropsychological Test Automated Battery (CANTAB).
\end{abstract}

This article discusses data derived from a study described in an article available at https://doi.org/10.1007/s40263-017-0443-y.

Electronic supplementary material The online version of this article (https://doi.org/10.1007/s40263-017-0487-z) contains supplementary material, which is available to authorized users.

David R. Coghill

david.coghill@unimelb.edu.au

1 Departments of Paediatrics and Psychiatry, Faculty of Medicine, Dentistry and Health Sciences, University of Melbourne, Melbourne, VIC 3010, Australia

2 Division of Neuroscience, University of Dundee, Dundee, UK

3 Department of Child and Adolescent Psychiatry and Psychotherapy, Central Institute of Mental Health, Medical Faculty Mannheim, University of Heidelberg, Mannheim, Germany

4 Shire, Lexington, MA, USA

5 Child and Adolescent Neuropsychiatry Unit, Department of Biomedical Sciences, University of Cagliari, Cagliari, Italy
Methods Participants aged 6-17 years received dose-optimised open-label lisdexamfetamine dimesylate $(30,50$ or $70 \mathrm{mg} /$ day) for 104 weeks. Cognition was assessed using four CANTAB tasks; Delayed Matching to Sample (DMS), Spatial Working Memory (SWM), Stop Signal Task (SST) and Reaction Time (RTI). Key and additional variables were pre-specified for each CANTAB task; groupwise mean percentage changes in key variables from baseline of $>5 \%$ were considered potentially clinically significant. Results All 314 enrolled participants received lisdexamfetamine dimesylate and were included in the safety population, and $191(60.8 \%)$ completed the study. No potentially clinically significant deteriorations from baseline were observed in any key CANTAB variable over the 2 years of the study. Based on predefined thresholds, potentially clinically significant improvements from baseline were observed at 6 months (DMS median reaction time, mean per cent change, $-6.6 \%$; SWM total betweensearch errors, $-22.8 \%$; SST stop signal reaction time, $-18.9 \%$ ), and at the last on-treatment assessment (DMS median reaction time, $-6.5 \%$; SWM total between-search errors, $-32.6 \%$; SST stop signal reaction time, $-25.7 \%$ ). Conclusions Lisdexamfetamine dimesylate treatment for 2 years was not associated with deterioration of cognitive function in children and adolescents with attention-deficit/ hyperactivity disorder. Although improvements in some cognitive measures were observed, lack of a control group makes interpretation of the findings difficult. Further studies of the impact of stimulants on cognition are required.

ClinicalTrials.gov identifier NCT01328756. 


\section{Key Points}

Lisdexamfetamine dimesylate is a stimulant used to treat children, adolescents and adults with attentiondeficit/hyperactivity disorder.

SPD489-404 was a 2-year safety study of lisdexamfetamine dimesylate in children and adolescents with attention-deficit/hyperactivity disorder in which cognitive function was assessed using the Cambridge Neuropsychological Test Automated Battery (CANTAB).

In this study, lisdexamfetamine dimesylate treatment was not found to be associated with cognitive impairment; possible improvements in some domains of cognitive function were observed.

\section{Introduction}

The prodrug stimulant lisdexamfetamine dimesylate (LDX) is indicated to treat attention-deficit/hyperactivity disorder (ADHD) in children, adolescents and adults [1]. The efficacy of LDX in relieving the symptoms of ADHD has been demonstrated in a series of pivotal randomised controlled trials in North America and Europe [2-5]. In addition, a large body of evidence from trials of at least 12 months' duration indicates that the safety and tolerability profile of LDX is similar to that of other stimulants in people with ADHD [1, 6, 7]. A rapid return of symptoms following LDX withdrawal was observed in a randomised withdrawal study, demonstrating the need for continued treatment to maintain efficacy [8]. SPD489-404 was the first 2-year open-label safety study of LDX in children and adolescents with ADHD [9]. Over the duration of the study, treatmentemergent adverse events were reported in line with expectations for the stimulant class of ADHD medication, with decreased appetite, weight decrease, insomnia and headache being among the most common. The incidence of these common treatment-emergent adverse events peaked early in the study and declined thereafter [9].

Attention-deficit/hyperactivity disorder is characterised by the symptoms of inattention and/or hyperactivity-impulsivity, and by functional impairment. Impaired cognitive development is also reported to be associated with ADHD [10-12]. The domains of cognitive function that are often impaired in ADHD include those relating to executive functions, such as impulse control and working memory, as well as non-executive functions, such as memory and reaction time [10]. There are little data available on the cognitive effects of extended treatment with psychostimulants. Following a literature review that identified associations between methylphenidate therapy and cognition and/or motivation, Kovshoff et al. conducted a semi-structured interview in a sample enriched for such experiences, including children and adolescents with ADHD, clinicians, carers and teachers. Respondents most commonly cited problems with attention/concentration, with patients describing 'zoning out' and 'a tendency to stare into space for long periods of time' [13]. To this end, the European Medicines Agency has advised that neurocognitive assessments should be considered standard practice in long-term studies of ADHD medications, and that special attention should be paid to identifying potential adverse cognitive effects [14].

The Cambridge Neuropsychological Test Automated Battery (CANTAB), developed at the University of Cambridge, UK, in the 1980s, is a computer-based cognitive assessment system consisting of a battery of neuropsychological tasks, administered via a touchscreen computer [15]. The CANTAB tasks focus on three cognitive domains: working memory and planning [tasks: Spatial Span, Spatial Working Memory (SWM), Spatial Planning], attention [tasks: Set Shifting, Reaction Time (RTI), Visual Search] and visuospatial memory [tasks: Pattern and Spatial Recognition, Delayed Matching to Sample (DMS), Paired Associate Learning, Stop Signal Task (SST)] [16]. The CANTAB tasks have a well-established sensitivity to a wide range of cognitive effects. In particular, results of a meta-analysis have shown that the CANTAB is sensitive to cognitive dysfunction in people with ADHD, as well as to the modulation of cognition by psychostimulants and noradrenaline reuptake inhibitors [17]. Here, we report the results of predefined investigations into the long-term effects of LDX on cognition, assessed using four CANTAB tasks [DMS, SWM, SST and RTI].

\section{Methods}

\subsection{Study Design and Conduct}

SPD489-404 was a 2-year, phase IV, open-label safety study of dose-optimised LDX in children and adolescents with ADHD (ClinicalTrials.gov identifier: NCT01328756). Full details of the study design, conduct, methods and outcomes have been published previously [9].

The study was conducted in accordance with current applicable international and national regulations and ethical requirements. Before participation, each patient's parent or legally authorised guardian provided informed consent, and each patient provided assent, if applicable. 
The study protocol was approved by an independent ethics committee/institutional review board and regulatory agency in each centre (as appropriate). The study took place at 35 sites in ten European countries (Belgium, Germany, Hungary, Italy, the Netherlands, Poland, Romania, Spain, Sweden and the UK) between July 2011 and September 2014.

\subsection{Participants}

Patients eligible to take part in the study were aged 6-17 years, were required to meet Diagnostic and Statistical Manual of Mental Disorders, Fourth Edition-Text Revision [18] criteria for a primary diagnosis of ADHD and had to have an ADHD Rating Scale IV (ADHD-RS-IV [19]) total score of $\geq 28$ at the baseline visit. Patients who had participated in one of the three previous LDX studies $[2,20,21]$ were excluded from the present study if they had discontinued the previous study because of protocol nonadherence or non-compliance, or if they had experienced a clinically significant treatment-emergent adverse event or adverse event that would preclude further exposure to LDX. Participants were also excluded if their current ADHD medication provided adequate control of ADHD symptoms with acceptable tolerability. Other inclusion and exclusion criteria have been reported previously [9].

Participants were not required to enter the present study immediately from any of the previous studies. However, patients with a gap of at least 7 days between exiting a previous study and entering this study were required to have a baseline ADHD-RS-IV total score of $\geq 28$ to be enrolled.

\subsection{Study Drug Administration}

Participants took once-daily morning doses of open-label LDX during the 4-week dose-optimisation period and the subsequent 100-week dose-maintenance period. During dose optimisation, participants' doses were actively titrated to LDX 30,50 or $70 \mathrm{mg} /$ day, starting at $30 \mathrm{mg} /$ day and increasing or decreasing in weekly 20-mg steps until an acceptable response was achieved. An acceptable response was defined as a reduction of $\geq 30 \%$ in ADHD-RS-IV total score from baseline with a Clinical Global ImpressionsImprovement [22] score of 1 or 2 and tolerable side effects. Dose adjustments were also permitted, if necessary, during the dose-maintenance period. All doses ranged from a minimum of $30 \mathrm{mg} /$ day to a maximum of $70 \mathrm{mg} /$ day.

\subsection{Neuropsychological Tasks and Testing}

Cognitive function was assessed at baseline (week 0), at weeks 4, 24, 48, 72 and 104 of the open-label treatment period and/or at the early termination visit (Fig. 1). Patients were also assessed at a subsequent safety follow-up visit (28-30 days following the last dose of LDX; data not presented). Four neuropsychological tasks from CANTAB were selected to assess cognition: DMS, SWM, SST and RTI (Table 1). DMS, SWM and SST were considered to be the most relevant tasks with which to capture the key cognitive domains implicated in the pathophysiology and treatment of ADHD [17, 23, 24], and the RTI task has been shown to be sensitive to potential deleterious effects [25]. Computerised tasks were administered using a touchscreen device.

\subsection{Neurocognitive Outcome Variables and Statistical Analysis}

Enrolment of about 300 patients was planned, but the sample size of this open-label uncontrolled study was not based on a statistical power calculation. The CANTAB results were analysed for the safety population, defined as all participants who received at least one dose of LDX during the study, regardless of whether or not they completed the study.

For each of the four selected CANTAB tasks, key and additional variables were pre-specified, based on recommendations from Cambridge Cognition Ltd (Cambridge, UK) (Table 1). Key variables were: per cent correct and median reaction time on correct trials for DMS, total between-search errors for SWM, stop signal reaction time for SST, and simple median reaction time and 5-choice median reaction time for RTI. The mean percentage change from baseline at each on-treatment assessment was calculated as the mean change from baseline divided by the mean at baseline in the safety population. No inferential hypotheses were tested statistically for any of the SPD489404 safety outcomes, including the CANTAB outcomes described here.

Given that there was no comparator arm in this study, a threshold for potential clinical significance was predefined as a groupwise change from baseline of $\pm 5 \%$ in key CANTAB variables. This threshold was based on previous experience with the CANTAB tasks and published data from a 4-year longitudinal study of cognition in 17 boys with ADHD and 17 age-matched typically developing boys [23]. Over 4 years, the mean per cent change from baseline on SWM between-search errors in untreated boys with ADHD was $40 \%$, equating to an improvement of $~ 5 \%$ per 6 months. Mean DMS per cent correct improved by $\sim 3 \%$ every 6 months. Stop Signal Task and RTI were not assessed in this study. 


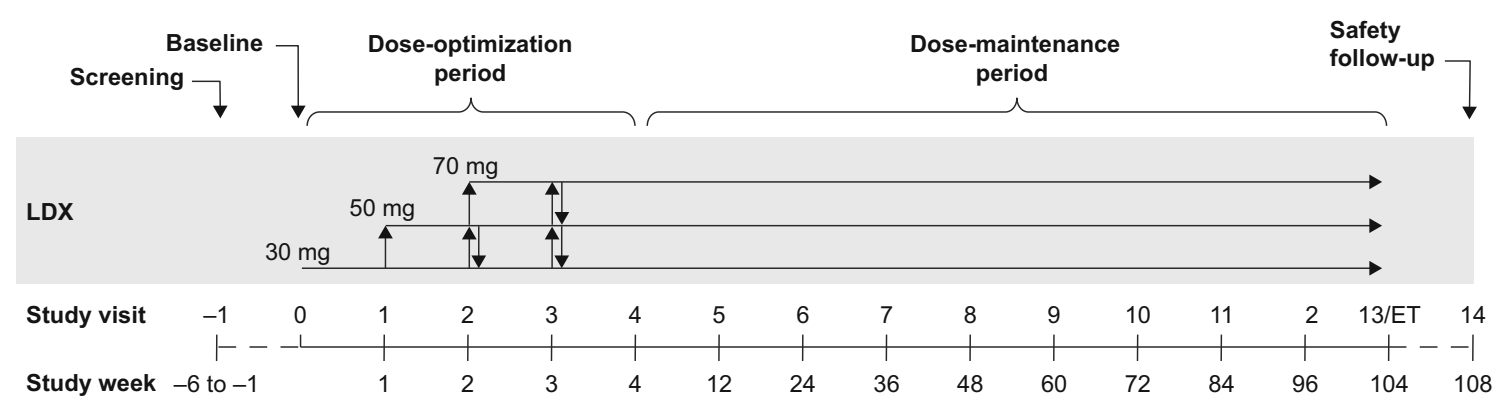

Fig. 1 SPD489-404 study design. Cambridge Neuropsychological Test Automated Battery (CANTAB) assessments were carried out at baseline (week 0), at weeks 4, 24, 48, 72 and 104 of the open-label

\section{Results}

\subsection{Patient Disposition and Characteristics}

Details of the study population have been published previously [9]. All 314 enrolled patients received at least one dose of LDX and were included in the safety population. Of these, 124 patients (39.5\%) had participated in a previous LDX study [2, 20, 21] and $191(60.8 \%)$ completed the present study. The most common reasons for study discontinuation were withdrawal by the participant $(41 / 314$ [13.1\%]) and adverse events (39/314 [12.4\%]). At baseline, the patients had a mean age of 11.4 years (standard deviation, 2.88 years), and 202/314 (64.3\%) were children aged 6-12 years. Most participants were boys (250/314 [79.6\%]) and nearly all were white (310/314 [98.7\%]). The mean ADHD-RS-IV total score at baseline was 41.1 (standard deviation, 7.03).

\subsection{Delayed Matching to Sample (DMS)}

No potentially clinically significant deterioration was seen in the key DMS variables of per cent correct and median reaction time on correct trials (i.e. a decrease in per cent correct or an increase in time, respectively) (Fig. 2). The mean DMS per cent correct remained close to the baseline level throughout the study and at the last on-treatment assessment (LOTA). The mean DMS per cent correct in all trials with a delay, as well as simultaneous trials and trials with a 0 - or 12-s delay, generally remained close to baseline levels at all subsequent study visits and at LOTA. In trials with a 4-s delay, the mean per cent correct at LOTA was below the baseline value.

For mean DMS median reaction time on correct trials, improvements predefined as being potentially clinically significant were seen after 6 months and maintained throughout the study. Improvements in median reaction time were more pronounced in simultaneous trials and in treatment period and/or at the early termination (ET) visit. $L D X$ lisdexamfetamine dimesylate

trials with a delay of $0 \mathrm{~s}$ than in the more difficult trials with delays of 4 or $12 \mathrm{~s}$.

\subsection{Spatial Working Memory (SWM)}

No potentially clinically significant deterioration (indicated by an increase in score) was observed in the SWM key variable of total between-search errors (Fig. 3). By 6 months, improvements in SWM total between-search errors exceeded the predefined threshold for potential clinical significance, with a mean decrease from baseline of $22.8 \%$. This improvement was maintained throughout the study and a $32.6 \%$ mean decrease from baseline was observed at LOTA. For the additional variables of between-search errors for trials of six or eight tokens, mean changes from baseline indicated improvements at weeks 4-104 and at LOTA. Between-search errors for trials of four tokens were close to zero and there was little room for improvement during the study.

\subsection{Stop Signal Task (SST)}

No potentially clinically significant deterioration (indicated by an increase in reaction time) was observed in the SST key variable of stop signal reaction time (Fig. 4). Potentially clinically significant improvements in stop signal reaction time were observed, with $18.9 \%$ and $25.7 \%$ mean decreases from baseline at 6 months and at LOTA, respectively. For the additional variable of reaction time standard deviation on 'go' trials, mean changes from baseline indicated improvement throughout the study.

\subsection{Reaction Time (RTI)}

No potentially clinically significant deterioration (indicated by an increase in reaction time) was observed in the key RTI variables of simple median reaction time and 5-choice median reaction time (Fig. 5). Both key variables remained close to baseline levels at all subsequent study visits and at 
Table 1 Cambridge Neuropsychological Task Automated Battery (CANTAB) tasks used in SPD489-404

\begin{tabular}{|c|c|c|c|c|}
\hline $\begin{array}{l}\text { CANTAB } \\
\text { task }\end{array}$ & Domain of cognition & Description & $\begin{array}{l}\text { Key } \\
\text { variables }\end{array}$ & Additional variables \\
\hline \multirow[t]{2}{*}{$\begin{array}{l}\text { Delayed } \\
\text { matching } \\
\text { to sample } \\
\text { (DMS) }\end{array}$} & \multirow[t]{2}{*}{$\begin{array}{l}\text { Recognition memory and short- } \\
\text { term visual memory }\end{array}$} & \multirow[t]{2}{*}{$\begin{array}{l}\text { The participant is shown a complex visual } \\
\text { pattern and must then select the matching } \\
\text { pattern from four similar patterns shown } \\
\text { simultaneously, or with a delay of } 0,4 \text { or } 12 \mathrm{~s} \\
\text { after the sample is concealed and the choices } \\
\text { are revealed }\end{array}$} & $\begin{array}{l}\text { Per cent } \\
\text { correct }^{\mathrm{a}}\end{array}$ & $\begin{array}{l}\text { Per cent correct } \\
\text { ( simultaneous) } \\
\text { Per cent correct ( } 0 \text {-s } \\
\text { delay) } \\
\text { Per cent correct (4-s } \\
\text { delay) } \\
\text { Per cent correct (12-s } \\
\text { delay) } \\
\text { Per cent correct (all trials } \\
\text { excluding simultaneous) }\end{array}$ \\
\hline & & & $\begin{array}{l}\text { Median } \\
\text { reaction } \\
\text { time on } \\
\text { correct } \\
\text { trials }^{\mathrm{b}}\end{array}$ & $\begin{array}{l}\text { Median reaction time on } \\
\text { correct trials } \\
\text { (simultaneous) } \\
\text { Median reaction time on } \\
\text { correct trials (0-s delay) } \\
\text { Median reaction time on } \\
\text { correct trials (4-s delay) } \\
\text { Median reaction time on } \\
\text { correct trials (12-s } \\
\text { delay) }\end{array}$ \\
\hline $\begin{array}{l}\text { Spatial } \\
\text { working } \\
\text { memory } \\
\text { (SWM) }\end{array}$ & $\begin{array}{l}\text { Retention and manipulation of } \\
\text { visuospatial information, as well } \\
\text { as some aspects of executive } \\
\text { function }\end{array}$ & $\begin{array}{l}\text { The participant is shown a number of square } \\
\text { 'boxes' and must find a blue token in each } \\
\text { box. The number of boxes increases from four } \\
\text { to six and then to eight; the colour and } \\
\text { position of the boxes change from trial to trial }\end{array}$ & $\begin{array}{l}\text { Total } \\
\text { between- } \\
\text { search } \\
\text { errors }\end{array}$ & $\begin{array}{l}\text { Between-search errors } \\
\text { (four tokens) } \\
\text { Between-search errors } \\
\quad \text { (six tokens) } \\
\text { Between-search errors } \\
\text { (eight tokens) }\end{array}$ \\
\hline $\begin{array}{l}\text { Stop signal } \\
\text { task (SST) }\end{array}$ & $\begin{array}{l}\text { Response inhibition and mental } \\
\text { processing speed }\end{array}$ & $\begin{array}{l}\text { The participant must respond to an arrow } \\
\text { pointing in one of two directions by pressing } \\
\text { the corresponding button, but must inhibit } \\
\text { their response if a sound is heard }\end{array}$ & $\begin{array}{l}\text { Stop signal } \\
\text { reaction } \\
\text { time }^{\mathrm{d}}\end{array}$ & $\begin{array}{l}\text { Reaction time SD on 'go' } \\
\text { trials }\end{array}$ \\
\hline \multirow[t]{2}{*}{$\begin{array}{l}\text { Reaction } \\
\text { time } \\
\text { (RTI) }\end{array}$} & \multirow[t]{2}{*}{$\begin{array}{l}\text { Motor and mental response speed } \\
\text { and some aspects of impulsivity }\end{array}$} & \multirow{2}{*}{$\begin{array}{l}\text { The participant must respond in one of a variety } \\
\text { of ways to the appearance of a yellow dot, } \\
\text { either in a single location or in one of five } \\
\text { possible locations. The participant is } \\
\text { encouraged to go faster on each series of trials }\end{array}$} & $\begin{array}{l}\text { Simple } \\
\text { median } \\
\text { reaction } \\
\text { time }^{\mathrm{e}}\end{array}$ & $\begin{array}{l}\text { Simple reaction time SD } \\
\text { Simple premature } \\
\text { response errors }\end{array}$ \\
\hline & & & $\begin{array}{l}\text { 5-choice } \\
\text { median } \\
\text { reaction } \\
\text { time }^{f}\end{array}$ & $\begin{array}{l}\text { 5-choice reaction time SD } \\
\text { 5-choice premature } \\
\text { response errors }\end{array}$ \\
\hline
\end{tabular}

SD standard deviation

${ }^{\text {a }}$ The proportion of assessed trials (\%) in which the participant responded correctly on their first attempt, for all trials (key variable) or for each of the four trial types (additional variables). Higher values indicate better cognitive function

${ }^{\mathrm{b}}$ The median reaction time in assessed trials in which the participant responded correctly, for all trial types (key variable) or for each of the four trial types (additional variables). Lower values indicate better cognitive function

${ }^{\mathrm{c}}$ The number of times the participant returned to a box in which a token had already been found, in all trials (key variable) or separately in trials with four, six or eight tokens (additional variables). Lower values indicate better cognitive function

'The time between 'go' and 'stop' stimuli at which the participant inhibited their response in 50\% of trials (key variable) and its SD (additional variable). Lower values indicate better cognitive function

${ }^{\mathrm{e}}$ In assessed trials with a single stimulus, the time between stimulus and correct response (key variable) and its SD (additional variable), and the number of trials in which the participant responded prematurely (additional variable). Lower values indicate better cognitive function

${ }^{\mathrm{f}}$ In assessed trials with a stimulus in one of five possible locations, the time between stimulus and correct response (key variable) and its SD (additional variable), and the number of trials in which the participant responded prematurely (additional variable). Lower values indicate better cognitive function 
LOTA. For the additional variable of simple premature response errors, small decreases from baseline were observed at weeks 4-104 and at LOTA, suggesting improvement. All other additional variables remained close to baseline levels throughout the study.

\section{Discussion}

In this 2-year open-label study, LDX was well tolerated in children and adolescents with ADHD, with a safety profile consistent with that established in previous studies of up to 12 months' duration; there were no new safety signals of concern $[1,9]$. No potentially clinically significant deteriorations from baseline were observed in any of the four CANTAB tasks. In fact, there was some evidence for potentially clinically significant improvements from baseline in certain cognitive domains. However, as discussed below, the lack of a comparator group limits interpretation of these data.

The EMA has recommended that neurocognitive measures should be included in long-term studies of ADHD medications. Several studies have demonstrated that individuals with ADHD can have impairments in the cognitive domains of response inhibition, working memory, processing speed and reaction time variability $[12,17,26]$. Furthermore, certain psychoactive agents are known to impair cognition, including cannabis and benzodiazepines $[27,28]$; indeed, CANTAB assessments demonstrated that cannabis users had significant impairments in the quality of decision making and executive planning compared with controls [27]. To this end, the primary goal of the CAN$\mathrm{TAB}$ assessments during this 2-year study was to detect any potential deterioration in cognitive function. The present study provides evidence that LDX treatment is not associated with the deterioration of cognitive function in children and adolescents with ADHD.

A potential positive impact of LDX on cognitive function was observed in the present study in attention and short-term visual memory (measured by the DMS task). A much larger improvement occurred in the domains of response inhibition (measured by the SST task) and working memory and strategy (measured by the SWM task). In the absence of a placebo control group, it is not possible to dissect drug-induced changes in performance in cognitive tasks from natural improvement over time, or improvement owing to practice effects. However, the speed of improvements in SST stop signal reaction time and SWM total between-search errors (mean changes of $14.9 \%$ and $-15.7 \%$ from baseline to week 4 , respectively) is more rapid and larger than would be expected in the absence of the drug in young people with ADHD [23], and also occurred in parallel with rapid symptomatic improvements during the first 4 weeks [9]. It is, however, unclear whether the cognitive improvements observed in this study caused the improvements in core ADHD symptoms, or were themselves secondary to ADHD symptomatic improvements (e.g. as a result of reduced symptoms of hyperactivity and inattention), or if these two aspects of functioning were independent of each other, as has been suggested by previous work [29].

The underlying mechanism notwithstanding, response inhibition is a key cognitive treatment target for ADHD, and the data presented showing a possible positive effect of LDX on this and other domains of executive function warrant further well-controlled investigations. Stratification by baseline demographics and clinical characteristics, as well as dose-response effects of LDX and the effects of discontinuation on CANTAB performance, are potential areas of interest for future investigation.

While the present study was the first to assess cognitive function in people receiving LDX, previous studies have investigated the effects of alternative ADHD medications on cognition. A meta-analysis of randomised controlled trials found that the stimulant methylphenidate was significantly more effective than placebo at improving cognitive function in children and adolescents with ADHD in acute challenge situations [30]. Methylphenidate treatment was associated with improvements across all five neuropsychological domains examined (executive memory, non-executive memory, reaction time, reaction time variability and response inhibition). However, effect sizes for neurocognitive outcomes were smaller than those reported for improvements in ADHD symptom scores and no studies of longer term methylphenidate use were included in the analysis [30]. The non-stimulant ADHD treatment atomoxetine has also been associated with improvements in some aspects of cognitive function in children and adolescents with ADHD, although the data are limited and again relate only to acute challenge [24, 31].

A number of key limitations should be considered when interpreting the findings presented here. First, the openlabel nature of the study and the lack of a control arm preclude firm conclusions about the potential beneficial effects of LDX. Change-from-baseline data are presented, but the lack of adequate normative developmental data for the CANTAB tasks across this age range makes it difficult to distinguish between potential treatment benefits and improvements relating to the normal development of children and adolescents over a 2-year period. In particular, a contribution of the natural developmental improvement in symptoms (especially hyperactive/impulsive symptoms) to the improvement in the SST stop signal reaction time task cannot be excluded. Second, although the four CANTAB tasks used in this study were selected to evaluate potential cognitive effects of stimulant treatment in 

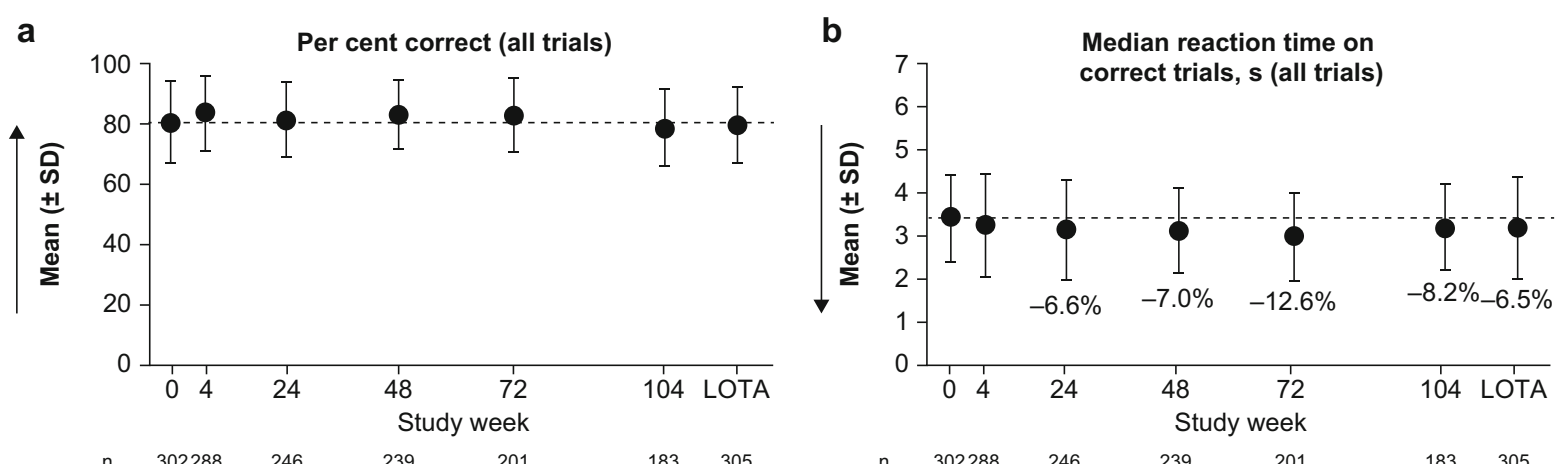

C

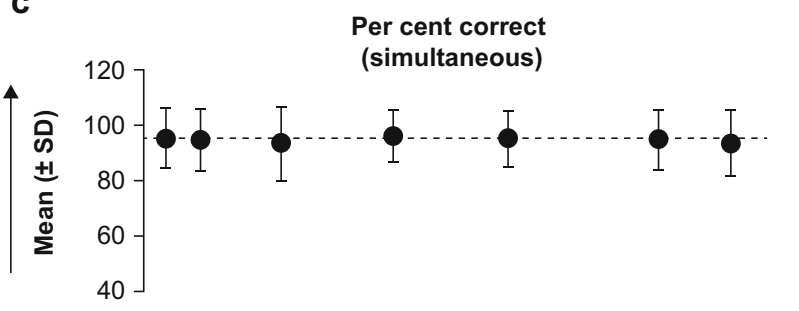

d

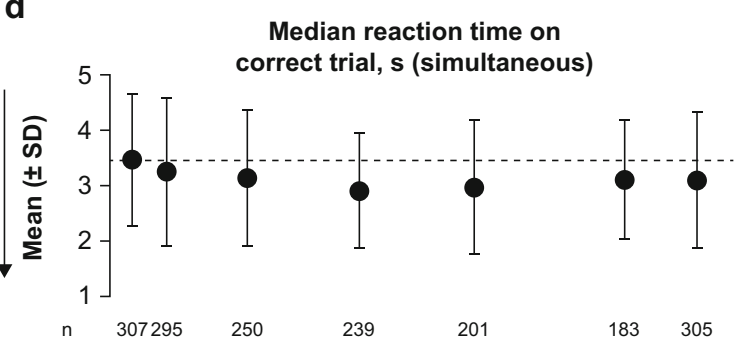

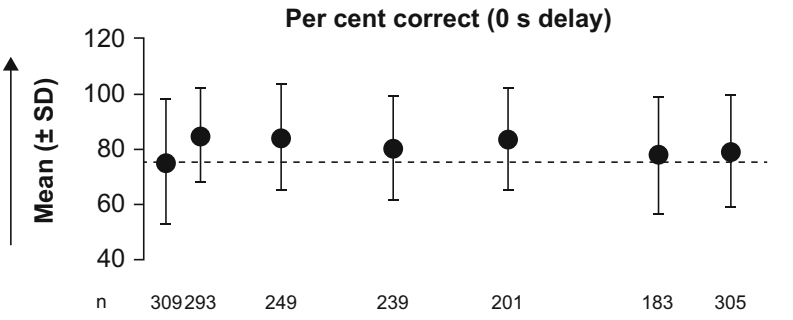
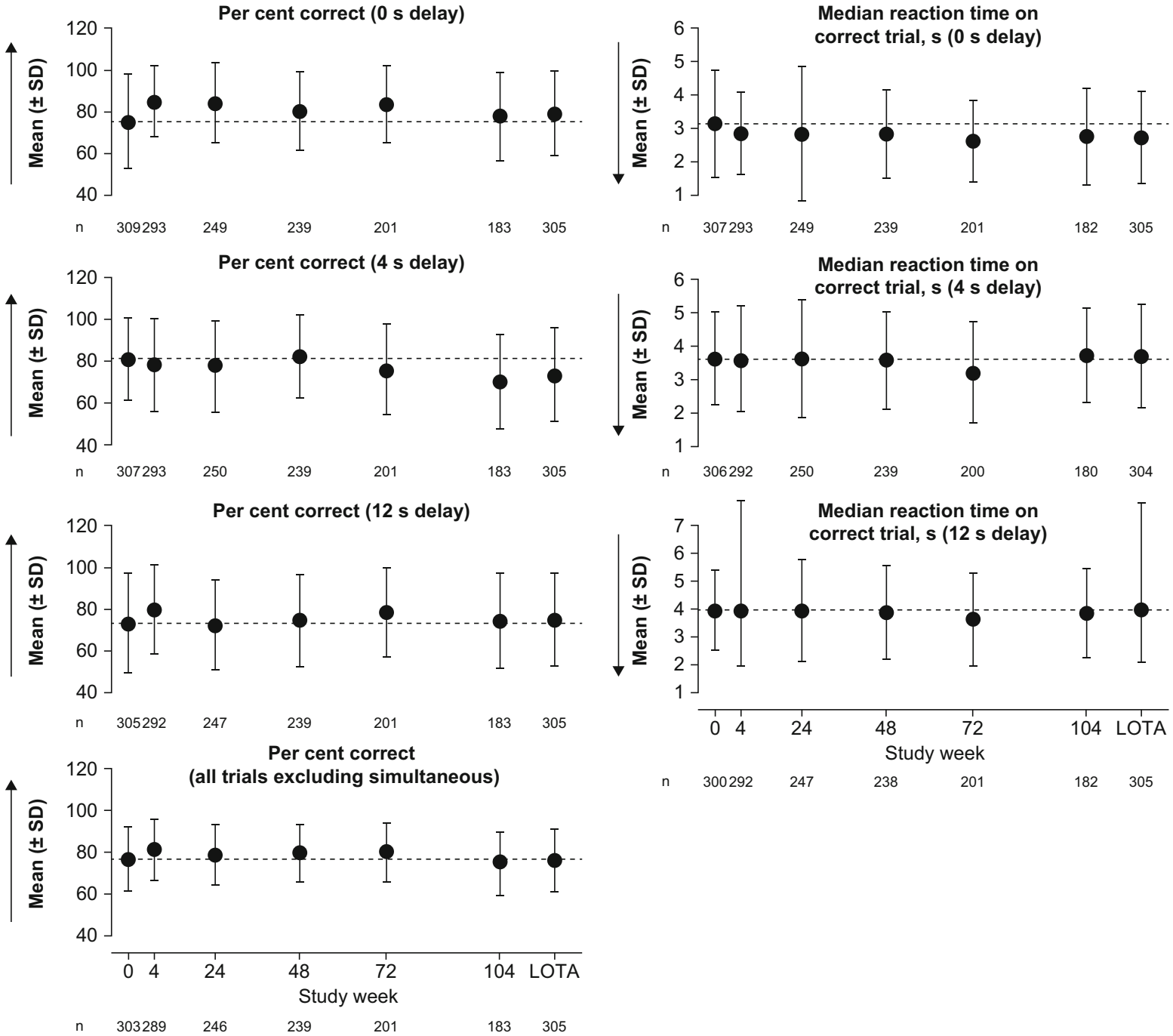
४Fig. 2 Delayed Matching to Sample outcomes: a, b key and c, d additional variables. Dashed lines indicate the mean at baseline (week 0). Arrows show the direction of improvement; higher per cent correct and lower median reaction time indicate better cognitive function. Annotated percentages indicate potentially clinically significant changes from baseline (key variables only; parts $\mathbf{a}$ and $\mathbf{b}$ ). Groupwise summary data are shown in Online Resource 1. LOTA last on-treatment assessment, $S D$ standard deviation

a

Total between search errors

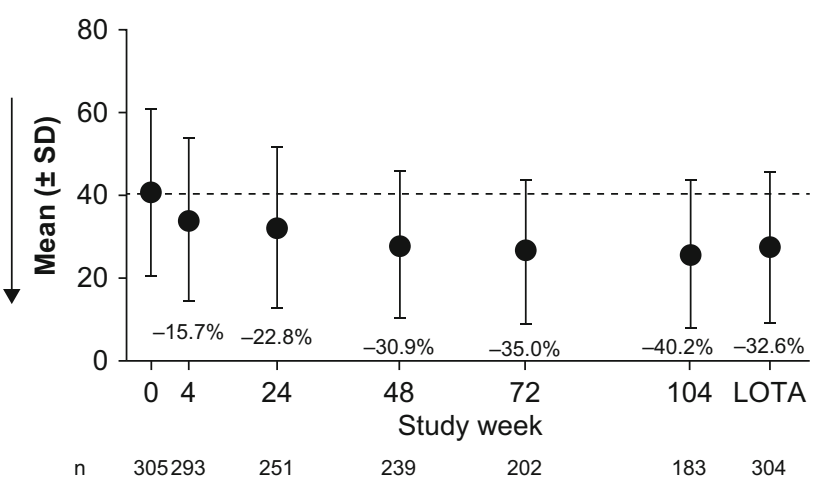

b
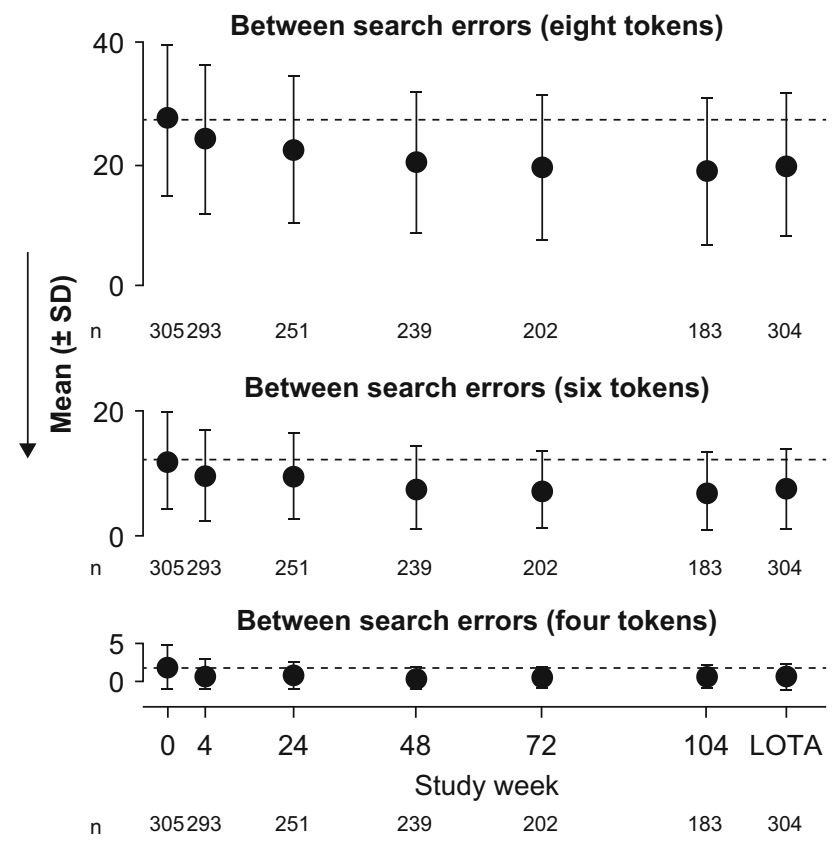

Fig. 3 Spatial Working Memory outcomes: a key and b additional variables. Dashed lines indicate the mean at baseline (week 0). Arrows show the direction of improvement; fewer between-search errors indicate better cognitive function. Annotated percentages indicate potentially clinically significant changes from baseline (key variable only; part a). Groupwise summary data are shown in Online Resource 2. LOTA last on-treatment assessment, $S D$ standard deviation a

Stop signal reaction time, ms

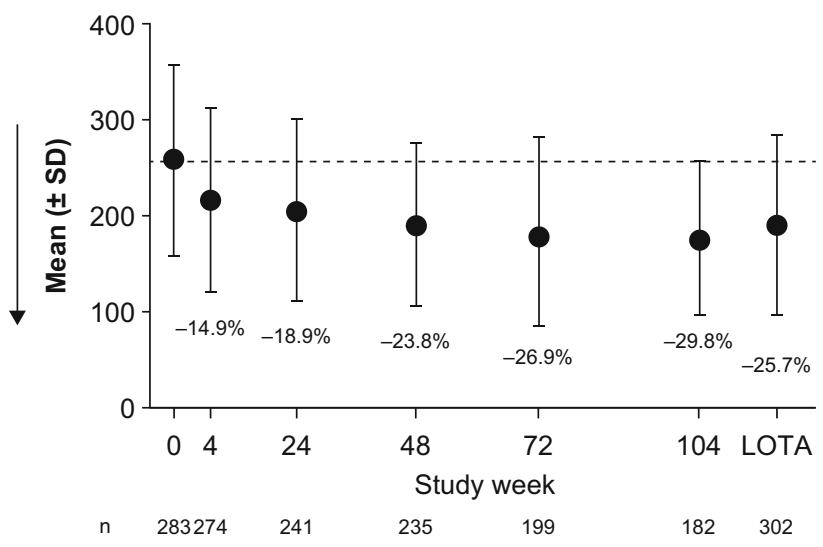

b

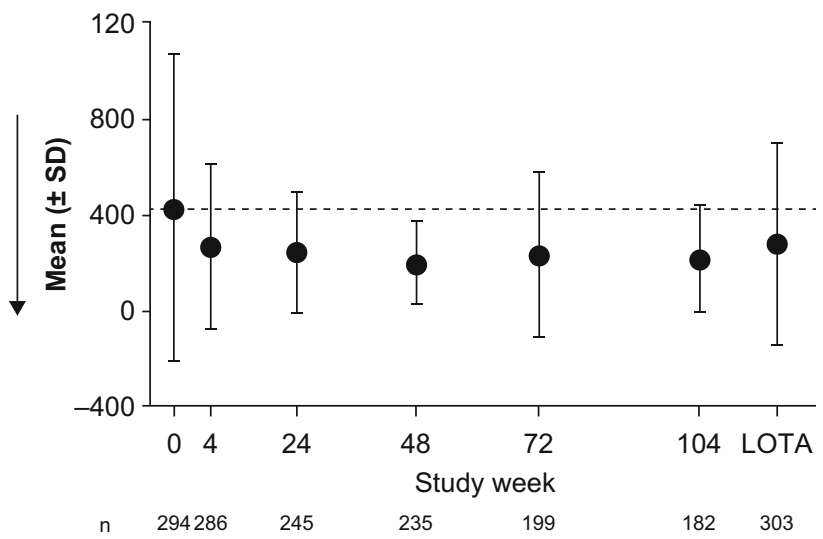

Fig. 4 Stop Signal Task outcomes: a key and $\mathbf{b}$ additional variables. Dashed lines indicate the mean at baseline (week 0). Arrows show the direction of improvement; lower reaction time and lower reaction time standard deviation (SD) on 'go' trials indicate better cognitive function. Annotated percentages indicate potentially clinically significant changes from baseline (key variable only; part a). Groupwise summary data are shown in Online Resource 3. LOTA last ontreatment assessment

children and adolescents with ADHD, LDX may have affected cognitive function in domains that were not tested. Third, because data were analysed at the group level only, potentially clinically significant changes in individual patients' cognitive function were not examined. Finally, the response rate of about $60 \%$ observed in this clinical trial setting is higher than might be expected in clinical practice, suggesting that the observed improvements in CANTAB may also exceed those seen routinely by physicians.

Important strengths of SPD489-404 are the 2-year duration, the inclusion of a large number of participants 
Simple median reaction time, $\mathrm{ms}$

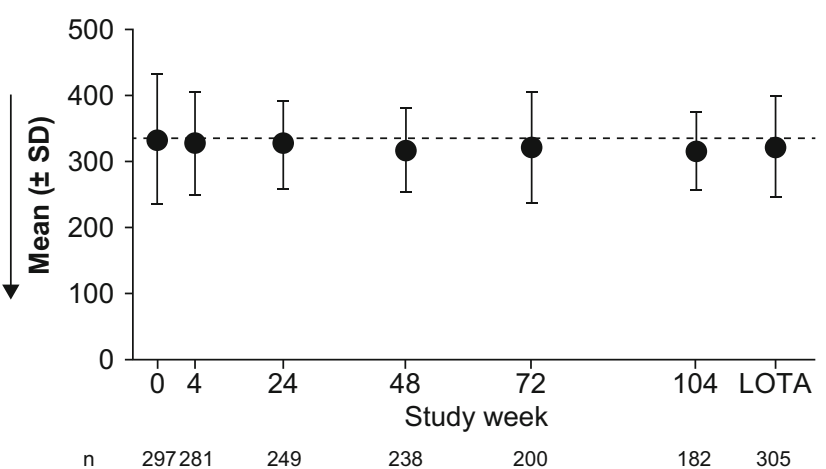

C
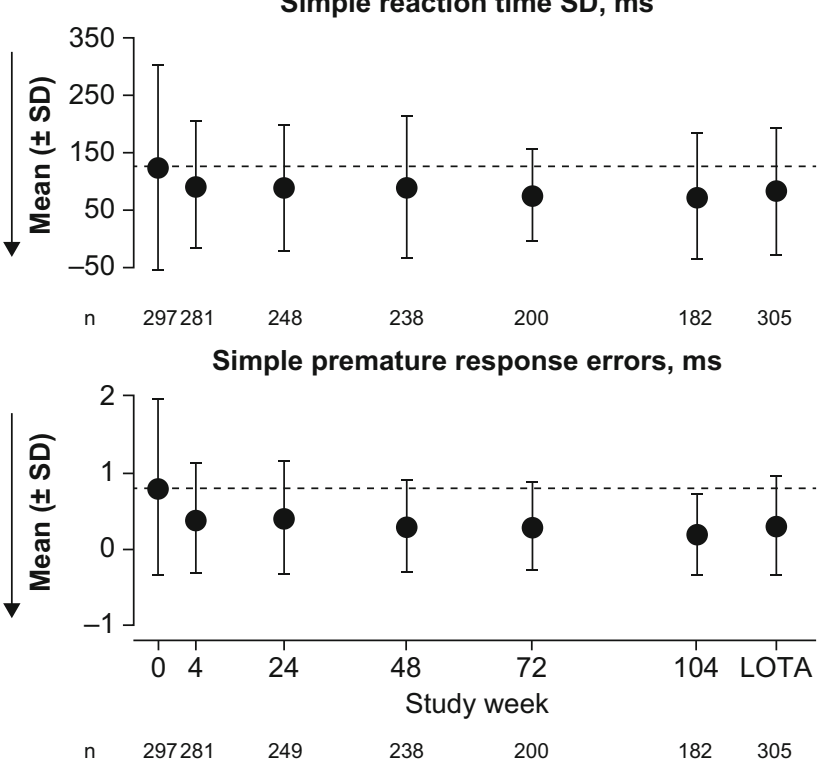

Fig. 5 Reaction Time outcomes: a, b key and c, d additional variables. Dashed lines indicate the mean at baseline (week 0). Arrows show the direction of improvement; lower reaction time, lower reaction time standard deviation (SD) and lower premature

from multiple centres in European countries and the inclusion of the CANTAB to assess cognition. The CANTAB tasks have proven congruence with traditional neuropsychological tasks and are an accepted and validated measure of cognitive function in patients with ADHD [32]. The CANTAB includes several different tasks with sensitivity to particular types of neurocognitive dysfunction. Furthermore, because the tasks are computerised, the CANTAB benefits from reliability of administration and practice effects are minimised by the use of parallel versions across all testing sessions. Additionally, the inclusion of multiple difficulty levels in the SWM and DMS allows any ceiling or floor effects to be identified [33]. b

5-choice median reaction time, $\mathrm{ms}$

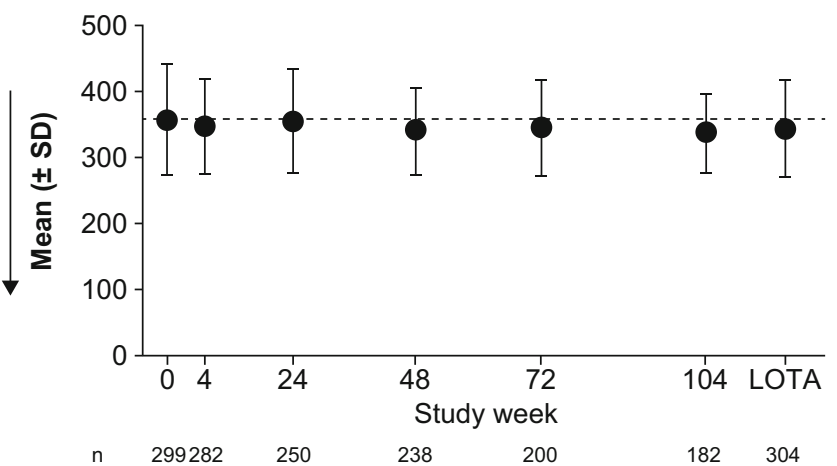

d
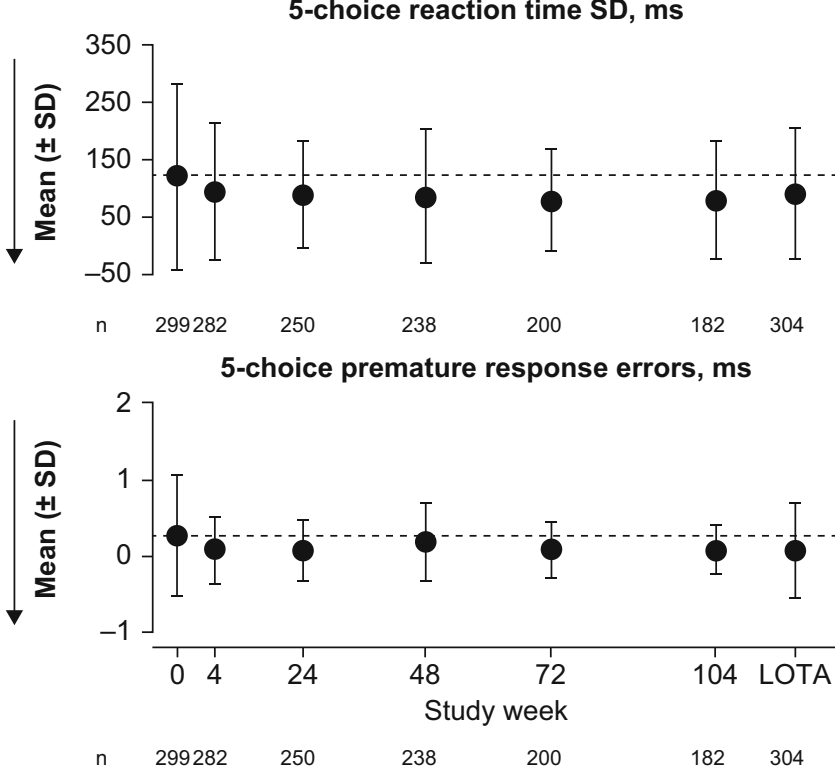

response errors indicate better cognitive function. No potentially clinically significant changes from baseline were identified (key variables only; parts a and b). Groupwise summary data are shown in Online Resource 4. LOTA last on-treatment assessment

\section{Conclusion}

This 2-year study provides evidence that LDX treatment is not associated with cognitive impairment in children and adolescents with ADHD, and suggests that potential longterm cognitive function improvements may be worthy of further investigation in well-controlled studies.

Acknowledgements We thank the patients and investigators who took part in this study. Under the direction of the authors and funded by Shire International GmbH, Dr. M. G. Cottingham and Dr. A. L. Jones of Oxford PharmaGenesis, Oxford, UK provided writing assistance for this publication. Editorial assistance in formatting, proofreading, copy editing and fact checking was also provided by 
Oxford PharmaGenesis. Although employees of the sponsor were involved in the design, collection, analysis, interpretation and fact checking of information, the content of this manuscript, the interpretation of the data and the decision to submit the manuscript for publication in CNS Drugs was made by the authors independently.

\section{Compliance with Ethical Standards}

Funding The study was funded by Shire Development LLC. Writing and editing assistance for this paper was provided by Oxford PharmaGenesis, funded by Shire International $\mathrm{GmbH}$. Shire International $\mathrm{GmbH}$ also funded open access.

Conflict of interest Caleb Bliss and Brigitte Robertson are employees of Shire and own stocks or stock options. The following authors have received compensation for serving as consultants or speakers for, or they or the institutions they work for have received research support or royalties from, the companies or organisations indicated: Tobias Banaschewski (Actelion, CIP Medien, Hexal Pharma, Hogrefe, Kohlhammer, Lilly, Lundbeck, Medice, Novartis, Oxford University Press, Shire and Vifor Pharma); David R. Coghill (Eli Lilly, Janssen-Cilag, Medice, Novartis, Oxford University Press, Shire and Vifor Pharma); Alessandro Zuddas (Angelini, EduPharma, Janssen, Lilly, Lundbeck, Oxford University Press, Roche, Servier and Shire).

Ethics approval All procedures performed in studies involving human participants were in accordance with the ethical standards of the institutional and/or national research committee and with the 1964 Helsinki Declaration and its later amendments or comparable ethical standards.

Informed consent Each patient's parent/legal guardian provided written informed consent, and assent was obtained from each participant (as applicable) before taking part in the study.

Open Access This article is distributed under the terms of the Creative Commons Attribution-NonCommercial 4.0 International License (http://creativecommons.org/licenses/by-nc/4.0/), which permits any noncommercial use, distribution, and reproduction in any medium, provided you give appropriate credit to the original author(s) and the source, provide a link to the Creative Commons license, and indicate if changes were made.

\section{References}

1. Coghill DR, Caballero B, Sorooshian S, Civil R. A systematic review of the safety of lisdexamfetamine dimesylate. CNS Drugs. 2014;28(6):497-511.

2. Coghill D, Banaschewski T, Lecendreux M, Soutullo C, Johnson M, Zuddas A, et al. European, randomized, phase 3 study of lisdexamfetamine dimesylate in children and adolescents with attention-deficit/hyperactivity disorder. Eur Neuropsychopharmacol. 2013;23(10):1208-18.

3. Biederman J, Krishnan S, Zhang Y, McGough JJ, Findling RL. Efficacy and tolerability of lisdexamfetamine dimesylate (NRP104) in children with attention-deficit/hyperactivity disorder: a phase III, multicenter, randomized, double-blind, forced-dose, parallel-group study. Clin Ther. 2007;29(3):450-63.

4. Findling RL, Childress AC, Cutler AJ, Gasior M, Hamdani M, Ferreira-Cornwell MC, et al. Efficacy and safety of lisdexamfetamine dimesylate in adolescents with attention-deficit/ hyperactivity disorder. J Am Acad Child Adolesc Psychiatry. 2011;50(4):395-405.

5. Adler LA, Goodman DW, Kollins SH, Weisler RH, Krishnan S, Zhang Y, et al. Double-blind, placebo-controlled study of the efficacy and safety of lisdexamfetamine dimesylate in adults with attention-deficit/hyperactivity disorder. J Clin Psychiatry. 2008;69(9):1364-73.

6. Findling RL, Childress AC, Krishnan S, McGough JJ. Long-term effectiveness and safety of lisdexamfetamine dimesylate in school-aged children with attention-deficit/hyperactivity disorder. CNS Spectr. 2008;13(7):614-20.

7. Weisler R, Young J, Mattingly G, Gao J, Squires L, Adler L, et al. Long-term safety and effectiveness of lisdexamfetamine dimesylate in adults with attention-deficit/hyperactivity disorder. CNS Spectr. 2009;14(10):573-85.

8. Coghill DR, Banaschewski T, Lecendreux M, Johnson M, Zuddas A, Anderson CS, et al. Maintenance of efficacy of lisdexamfetamine dimesylate in children and adolescents with attentiondeficit/hyperactivity disorder: randomized-withdrawal study design. J Am Acad Child Adolesc Psychiatry. 2014;53(6):647.e1-657.e1.

9. Coghill D, Banaschewski T, Nagy P, Hernández Otero I, Soutullo C, Yan B, et al. Long-term safety and efficacy of lisdexamfetamine dimesylate in children and adolescents with ADHD: a phase IV, 2-year open-label study in Europe. CNS Drugs. 2017;31(7):625-38.

10. Coghill DR, Seth S, Matthews K. A comprehensive assessment of memory, delay aversion, timing, inhibition, decision making and variability in attention deficit hyperactivity disorder: advancing beyond the three-pathway models. Psychol Med. 2014;44(9):1989-2001.

11. Rhodes SM, Coghill DR, Matthews K. Neuropsychological functioning in stimulant-naive boys with hyperkinetic disorder. Psychol Med. 2005;35(8):1109-20.

12. Lipszyc J, Schachar R. Inhibitory control and psychopathology: a meta-analysis of studies using the stop signal task. J Int Neuropsychol Soc. 2010;16(6):1064-76.

13. Kovshoff H, Banaschewski T, Buitelaar JK, Carucci S, Coghill D, Danckaerts M, et al. Reports of perceived adverse events of stimulant medication on cognition, motivation, and mood: qualitative investigation and the generation of items for the Medication and Cognition Rating Scale. J Child Adolesc Psychopharmacol. 2016;26(6):537-47.

14. European Medicines Agency. Guideline on the clinical investigation of medicinal products for the treatment of attention-deficit/ hyperactivity disorder (ADHD). 2010. http://www.ema.europa. eu/docs/en_GB/document_library/Scientific_guideline/2010/08/ WC500095686.pdf. Accessed 11 Jan 2018.

15. Sahakian BJ, Morris RG, Evenden JL, Heald A, Levy R, Philpot $\mathrm{M}$, et al. A comparative study of visuospatial memory and learning in Alzheimer-type dementia and Parkinson's disease. Brain. 1988;111(Pt 3):695-718.

16. Wild K, Howieson D, Webbe F, Seelye A, Kaye J. Status of computerized cognitive testing in aging: a systematic review. Alzheimers Dement. 2008;4(6):428-37.

17. Chamberlain SR, Robbins TW, Winder-Rhodes S, Muller U, Sahakian BJ, Blackwell AD, et al. Translational approaches to frontostriatal dysfunction in attention-deficit/hyperactivity disorder using a computerized neuropsychological battery. Biol Psychiatry. 2011;69(12):1192-203.

18. American Psychiatric Association. Diagnostic and statistical manual of mental disorders. 4th ed. Washington, DC: American Psychiatric Association; 2000 (text revision).

19. DuPaul GJ, Power TJ, Anastopoulos AD, Reid R. ADHD Rating Scale-IV: checklists, norms, and clinical interpretation. New York: Guilford Press; 1998. 
20. Coghill DR, Banaschewski T, Lecendreux M, Zuddas A, Dittmann RW, Otero IH, et al. Efficacy of lisdexamfetamine dimesylate throughout the day in children and adolescents with attention-deficit/hyperactivity disorder: results from a randomized, controlled trial. Eur Child Adolesc Psychiatry. 2014;23(2):61-8.

21. Dittmann RW, Cardo E, Nagy P, Anderson CS, Bloomfield R, Caballero B, et al. Efficacy and safety of lisdexamfetamine dimesylate and atomoxetine in the treatment of attention-deficit/ hyperactivity disorder: a head-to-head, randomized, double-blind, phase IIIb study. CNS Drugs. 2013;27(12):1081-92.

22. Guy W. Clinical global impression scale. ECDEU Assess Man Psychopharmacol-Revis Vol DHEW Publ No ADM. 1976;76(338):218-22.

23. Coghill DR, Hayward D, Rhodes SM, Grimmer C, Matthews K. A longitudinal examination of neuropsychological and clinical functioning in boys with attention deficit hyperactivity disorder (ADHD): improvements in executive functioning do not explain clinical improvement. Psychol Med. 2014;44(5):1087-99.

24. Gau SS, Shang CY. Improvement of executive functions in boys with attention deficit hyperactivity disorder: an open-label follow-up study with once-daily atomoxetine. Int J Neuropsychopharmacol. 2010;13(2):243-56.

25. Jakala P, Riekkinen M, Sirvio J, Koivisto E, Riekkinen P Jr. Clonidine, but not guanfacine, impairs choice reaction time performance in young healthy volunteers. Neuropsychopharmacology. 1999;21(4):495-502.

26. Bilder RM, Loo SK, McGough JJ, Whelan F, Hellemann G, Sugar C, et al. Cognitive effects of stimulant, guanfacine, and combined treatment in child and adolescent attention-deficit/hyperactivity disorder. J Am Acad Child Adolesc Psychiatry. 2016;55(8):667-73.
27. Grant JE, Chamberlain SR, Schreiber L, Odlaug BL. Neuropsychological deficits associated with cannabis use in young adults. Drug Alcohol Depend. 2012;121(1-2):159-62.

28. Robbins TW, Semple J, Kumar R, Truman MI, Shorter J, Ferraro A, et al. Effects of scopolamine on delayed-matching-to-sample and paired associates tests of visual memory and learning in human subjects: comparison with diazepam and implications for dementia. Psychopharmacology (Berl). 1997;134(1):95-106.

29. Coghill DR, Rhodes SM, Matthews K. The neuropsychological effects of chronic methylphenidate on drug-naive boys with attention-deficit/hyperactivity disorder. Biol Psychiatry. 2007;62(9):954-62.

30. Coghill DR, Seth S, Pedroso S, Usala T, Currie J, Gagliano A. Effects of methylphenidate on cognitive functions in children and adolescents with attention-deficit/hyperactivity disorder: evidence from a systematic review and a meta-analysis. Biol Psychiatry. 2014;76(8):603-15.

31. Shang CY, Gau SS. Improving visual memory, attention, and school function with atomoxetine in boys with attention-deficit/ hyperactivity disorder. J Child Adolesc Psychopharmacol. 2012;22(5):353-63.

32. Fried R, Hirshfeld-Becker D, Petty C, Batchelder H, Biederman J. How informative is the CANTAB to assess executive functioning in children with ADHD? A controlled study. J Atten Disord. 2015;19(6):468-75.

33. Soares FC, de Oliveira TC, de Macedo LD, Tomas AM, PicancoDiniz DL, Bento-Torres J, et al. CANTAB object recognition and language tests to detect aging cognitive decline: an exploratory comparative study. Clin Interv Aging. 2015;10:37-48. 